\title{
Complete genome of Rhizobium leguminosarum Norway, an ineffective Lotus micro-symbiont
}

\author{
Juan Liang ${ }^{\dagger}$, Anne Hoffrichter ${ }^{\dagger}$, Andreas Brachmann and Macarena Marín * (D)
}

\begin{abstract}
Rhizobia bacteria engage in nitrogen-fixing root nodule symbiosis, a mutualistic interaction with legume plants in which a bidirectional nutrient exchange takes place. Occasionally, this interaction is suboptimal resulting in the formation of ineffective nodules in which little or no atmospheric nitrogen fixation occurs. Rhizobium leguminosarum Norway induces ineffective nodules in a wide range of Lotus hosts. To investigate the basis of this phenotype, we sequenced the complete genome of $R /$ Norway and compared it to the genome of the closely related strain $R$. leguminosarum bv. viciae 3841. The genome comprises $7,788,085 \mathrm{bp}$, distributed on a circular chromosome containing $63 \%$ of the genomic information and five large circular plasmids. The functionally classified bacterial gene set is distributed evenly among all replicons. All symbiotic genes (nod, fix, nif) are located on the pRLN3 plasmid. Whole genome comparisons revealed differences in the metabolic repertoire and in protein secretion systems, but not in classical symbiotic genes.
\end{abstract}

Keywords: Symbiosis, Rhizobium, Legume, Ineffective nodulation, Genome

\section{Introduction}

Legume crops are central to sustainable agricultural practices and food security $[1,2]$. They have a low need for synthetic nitrogen fertilizers input, as they engage in a symbiosis with a group of diazotrophic bacteria collectively known as rhizobia. This symbiotic interaction is initiated by a molecular crosstalk between rhizobia and their cognate legume host. Upon recognition of specific signals, legume plants intracellularly accommodate rhizobia inside root organs called nodules, where they engage in a bidirectional nutrient exchange [3]. Occasionally, suboptimal interactions establish between the symbiotic partners. These lead to the formation of ineffective nodules in which limited to no nitrogen fixation occurs. These ineffective symbiotic associations are characterized by the formation of small white nodules, which results in reduced or no plant growth promotion [4].

Ineffective nitrogen-fixing symbioses have been described after introduction of crop legumes into areas where previously native legumes grew. The soil microbiota

\footnotetext{
* Correspondence: m.marin@biologie.uni-muenchen.de

${ }^{\dagger} J$ uan Liang and Anne Hoffrichter contributed equally to this work.

Institute of Genetics, Faculty of Biology, Ludwig-Maximilians-University

Munich, Munich, Germany
}

associated to native species can often outcompete inoculant strains [5]. For instance, ineffective nitrogen fixation occurs in fields where perennial and annual clovers co-exist $[6,7]$. In field trials, inoculant strains were unable to completely overcome indigenous $R$. leguminosarum bv. trifolii strains and occupied on average $50 \%$ of the nodules [8]. In extreme cases, it has been shown that endogenous rhizobia can completely block the nodulation of introduced rhizobia. For example, the nodulation of pea cultivars Afghanistan and Iran by rhizobial inoculants is suppressed in natural soils by the presence of a non-nodulating strain [9]. However, although ineffective nodulation is a limiting factor for sustainable agriculture, the molecular basis underlying it remains largely unknown [10].

Rhizobium leguminosarum $(R l)$ strains are cognate micro-symbionts of legumes, including Pisum, Lens, Lathyrus, Vicia, Phaseolus and Trifolium [11]. However, a $R$. leguminosarum strain isolated from a Lotus corniculatus nodule in Norway exhibits a different compatibility range that includes several Lotus species and ecotypes. $R l$ Norway does not induce effective nodules in any Lotus species tested so far [12]. Strikingly, there are host genotype specific differences in the nodulation phenotypes 
induced by $R l$ Norway, as it cannot induce nodules on $L$. japonicus Gifu, but induces bumps on L. japonicus Nepal, and white nodules on $L$. burttii and L. japonicus MG-20. This is in contrast to compatible Mesorhizobium strains that induce monomorphic phenotypes in the same plant ecotypes [12].

The striking diversity of ineffective nodulation phenotypes induced by $R l$ Norway in Lotus motivated us to sequence and annotate its complete genome, and to compare it to the published genome of $R$. leguminosarum bv. viciae 3841 ( $R l v$ 3841), a well-characterised $R$. leguminosarum strain. Here, we show that the genomes are largely conserved. There are no major differences in the nif and fix clusters required for nitrogen fixation and in the nod cluster essential for the production of nodulation factor. However, differences were observed in terms of metabolic and protein secretion system genes.

\section{Organism information}

\section{Classification and features}

$R l$ Norway is a Gram-negative strain in the order $R h i$ zobiales of the class Alphaproteobacteria (Table 1). Cells are rod-shaped and have dimensions of $0.84 \pm$ $0.11 \mu \mathrm{m}$ in width and $1.43 \pm 0.31 \mu \mathrm{m}$ in length (Fig. 1a). This strain is fast growing and forms colonies after 3 days in TY medium at $28^{\circ} \mathrm{C}$. Colonies on TY are circular and slightly domed, their surface is shiny and smooth, and their texture is moderately mucoid (Fig. 1b).

The phylogenetic relationship of $R l$ Norway was inferred based on a concatenated tree of the dnaK, $r e c A$, and $r p o B$ house-keeping genes (Fig. 2). Based on this phylogeny $R l$ Norway is placed within the $R$. leguminosarum group. The $16 \mathrm{~S}$ rRNA gene of $R l$ Norway shows more than $99.9 \%$ identity with its orthologs in other $R$. leguminosarum strains, such as

Table 1 Classification and general features of R/ Norway in accordance to the MIGS recommendations [46] published by the Genome Standards Consortium [47]

\begin{tabular}{|c|c|c|c|}
\hline MIGS ID & Property & Term & Evidence code $^{a}$ \\
\hline & Classification & Domain Bacteria & TAS [48] \\
\hline & & Phylum Proteobacteria & TAS [49] \\
\hline & & Class Alphaproteobacteria & $\operatorname{TAS}[50,51]$ \\
\hline & & Order Rhizobiales & TAS $[50,52]$ \\
\hline & & Family Rhizobiaceae & TAS [53-55] \\
\hline & & Genus Rhizobium & TAS [55-57] \\
\hline & & Species Rhizobium leguminosarum & TAS $[55,57-59]$ \\
\hline & Gram stain & Negative & IDA \\
\hline & Cell shape & Rod & IDA \\
\hline & Motility & Motile & IDA \\
\hline & Sporulation & Non-sporulating & NAS \\
\hline & Temperature range & Mesophile & NAS \\
\hline & Optimum temperature & $28^{\circ} \mathrm{C}$ & NAS \\
\hline & pH range; Optimum & Not reported & \\
\hline & Carbon source & Carbon sources sustaining growth are indicated in Figure S1 & IDA \\
\hline MIGS-6 & Habitat & Soil, root nodule of Lotus corniculatus & TAS [12] \\
\hline MIGS-6.3 & Salinity & Not reported & \\
\hline MIGS-22 & Oxygen requirement & Aerobic & NAS \\
\hline MIGS-15 & Biotic relationship & Free-living/symbiont & TAS [12] \\
\hline MIGS-14 & Pathogenicity & Non-pathogen & NAS \\
\hline MIGS-4 & Geographic location & Norway & TAS [12] \\
\hline MIGS-5 & Sample collection & 17. August 2006 & TAS [12] \\
\hline MIGS-4.1 & Latitude & $61^{\circ} 10^{\prime} 54.6^{\prime \prime}$ & TAS [12] \\
\hline MIGS-4.2 & Longitude & $08^{\circ} 57^{\prime} 54.5^{\prime \prime}$ & TAS [12] \\
\hline MIGS-4.4 & Altitude & Not available & \\
\hline
\end{tabular}

${ }^{a}$ Evidence codes - IDA Inferred from Direct Assay, TAS Traceable Author Statement (i.e., a direct report exists in the literature), NAS Non-traceable Author Statement (i.e., not directly observed for the living, isolated sample, but based on a generally accepted property for the species, or anecdotal evidence). These evidence codes are from the Gene Ontology project [60] 

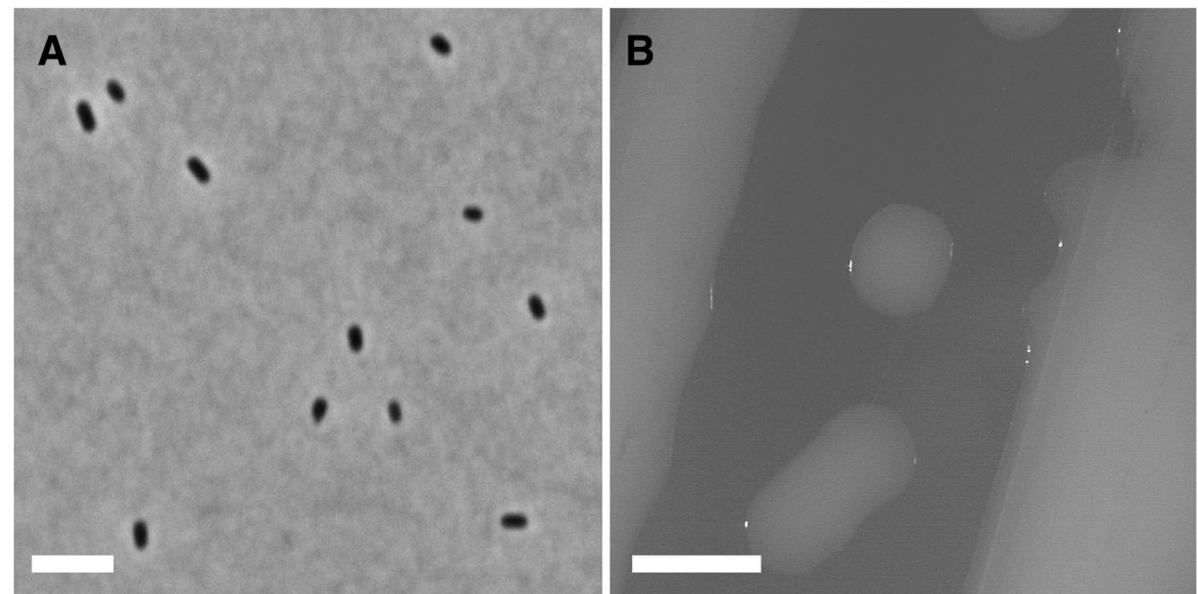

Fig. 1 Morphological characterisation of R/ Norway. a Phase contrast micrograph of $R /$ Norway grown in liquid TY medium. Scale bar: $1 \mu \mathrm{m}$. b Photomicrograph of the colony morphology of $R /$ Norway grown on TY medium. Scale bar: $1 \mathrm{~mm}$

$R l v 3841$ and $R l$ biovar trifolii WSM1325, WSM2304, and CB782.

The metabolic fingerprinting of $R l$ Norway was conducted with the Biolog GN2 MicroPlate. Rl Norway grew in multiple organic compounds as sole carbon source, these included Adonitol, L-Arabinose, D-Arabitol, D-Cellobiose, D-Fructose, and Glycerol, among others (Additional file 1: Figure S1). The metabolic fingerprinting of this strain was similar to the pattern described for other $R$. leguminosarum strains, but it was clearly distinct from the pattern of Rlv 3841 (Additional file 1: Figure S1) [13].

\section{Symbiotaxonomy}

$R l$ Norway was originally co-isolated from a $L$. corniculatus nodule together with two Mesorhizobium strains, but does not induce nodules in L. corniculatus or L. japonicus
Gifu, when inoculated alone [12]. However, it induces bumps on L. japonicus Nepal, and ineffective nodules on L. burttii and L. japonicus MG-20 [12]. This polymorphic nodulation phenotype is not observed, when these hosts are inoculated with Mesorhizobium strains [12]. $R l$ Norway induces ineffective nodules in Pisum, and Latyrus. The nodulation and symbiotic characteristics of $R l$ Norway are summarized in Additional file 2: Table S1.

\section{Genome sequencing information}

Genome project history

$R l$ Norway was selected for sequencing, because of the striking diversity of ineffective nodulation phenotypes that it induces in Lotus, a host that belongs to a different cross-inoculation group. The complete genome sequencing was performed at the Genomics Service Unit (LMU Biocenter, Munich). The nucleotide sequences reported

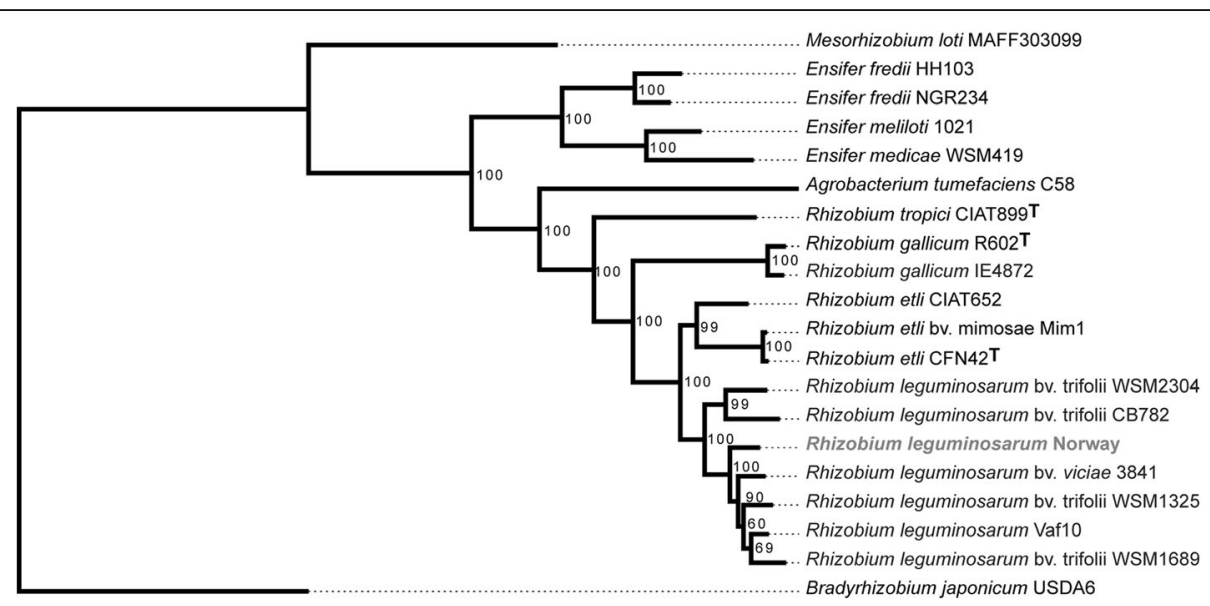

Fig. 2 Phylogenetic tree showing the relationship between R/ Norway and other Rhizobia. The tree was constructed by maximum likelihood using the concatenated sequences of recA, dnaK, and rpoB. The calculated bootstrap values are indicated at the nodes. R/ Norway is highlighted in bold grey. Type strains are indicated with superscript ${ }^{\top}$. B. japonicum USDA6 was used as an out-group 
in this study have been deposited in the GenBank database under accession numbers CP025012.1, CP025013.1, CP025014.1, CP025015.1, CP025016.1, and CP025017.1. The data is summarized in Table 2.

\section{Growth conditions and genomic DNA preparation}

$R l$ Norway was grown at $28{ }^{\circ} \mathrm{C}$ and $180 \mathrm{rpm}$ for 2 days in TY medium. Genomic DNA was isolated from $30 \mathrm{ml}$ of a bacterial suspension $\left(\mathrm{OD}_{600}=1.0\right)$ using the $\mathrm{CTAB}$ method [14]. The DNA quality was determined by nanodrop and gel electrophoresis.

\section{Genome sequencing and assembly}

The genome was sequenced using a combination of Illumina and MinION sequencing technologies. Library construction and sequencing were performed at the Genomics Service Unit (LMU Biocenter, Munich). For whole genome sequencing a short read DNA library was generated with the Nextera Kit (Illumina) according to manufacturer's instructions. Sequencing $(2 \times 150 \mathrm{bp}$, v2 chemistry) was performed on a MiSeq sequencer (Illumina) yielding around 15 Mio paired reads and $2.3 \mathrm{~Gb}$ of primary sequence. A long read library was prepared with the 1D Genomic DNA Sequencing Kit (Oxford Nanopores) according to manufacturer's instructions. MinION (Oxford Nanopores) sequencing resulted in around 180,000 sequences with a total of $670 \mathrm{Mb}$ primary sequence (mean length $3.8 \mathrm{~kb}$ ). Hybrid genome assembly with Unicycler v0.4.0 [15] using default settings resulted in six circular contigs. The average base coverage of the genome is $380 x$.

\section{Genome annotation}

Genome annotation was performed with RAST 2.0 [16, 17] and MicroScope [18]. Clusters of orthologous groups (COGs) of proteins were predicted using the COGNiTOR software [19], signal peptides were detected using the SignalP 4.1 server [20], and Pfam domains were predicted using the Pfam batch sequence search from EMBL-EBI [21]. Transmembrane predictions and CRISPR repeats were determined using the TMHMM Server v. 2.0 [22] and CRISPRFinder [23], respectively. All genes discussed in the text were manually inspected.

\section{Genome properties}

The genome of $R l$ Norway consists of 7,788,085 bp, distributed on a circular chromosome containing $63 \%$ of the genomic information and five large circular plasmids ranging from 280 to $1098 \mathrm{~kb}$ (Fig. 3). The complete genome and the chromosome are comparable in size to other $R$. leguminosarum strains $[13,24]$. The chromosome contains three identical rRNA operons and 54 tRNA genes, none of which are found on any of the five plasmids (Table 3 and Fig. 3). In total 7866 protein-encoding genes were identified. BUSCO analysis [25] confirmed complete presence of the core bacteria dataset. The six replicons have a comparable mix of functional classes (Additional file 3: Figure S2A). However, all genes from the BUSCO core bacteria dataset are located on the chromosome, with only a few additional gene duplications on the plasmid replicons.

\section{Insights from the genome sequence Extended insights}

The genomes of $R l$ Norway and $R l v 3841$ have a very similar relative occurrence of functional protein encoding genes (Additional file 3: Figure S2B) and do not show any gross genomic alterations. Interestingly, although $R l$ Norway contains more protein encoding genes than $R l v$ 3841 (7866 vs. 7263 genes), the number of genes for which a functional annotation could be retrieved is almost identical (6106 vs. 6105 genes). Hence, the major difference

Table 2 Genome sequencing project information for R/ Norway

\begin{tabular}{lll}
\hline MIGS ID & Property & Term \\
\hline MIGS 31 & Finishing quality & Finished \\
MIGS-28 & Libraries used & Paired-end (Illumina); 1D Genomic (Nanopore) \\
MIGS 29 & Sequencing platforms & Illumina MiSeq; Nanopore MinION \\
MIGS 31.2 & Fold coverage & $380 \times$ \\
MIGS 30 & Assemblers & Unicycler v0.4.0 \\
MIGS 32 & Gene calling method & MicroScope \\
& Locus Tag & CUJ84 \\
& Genbank ID & CP025012.1, CP025013.1, CP025014.1, CP025015.1, \\
& & CP025016.1, and CP025017.1 \\
& GenBank Date of Release & 31. January 2018 \\
MIGS 13 & BIOPROJECT & PRJNA417364 \\
& Project relevance & Agriculture, root nodule symbiosis \\
\hline
\end{tabular}




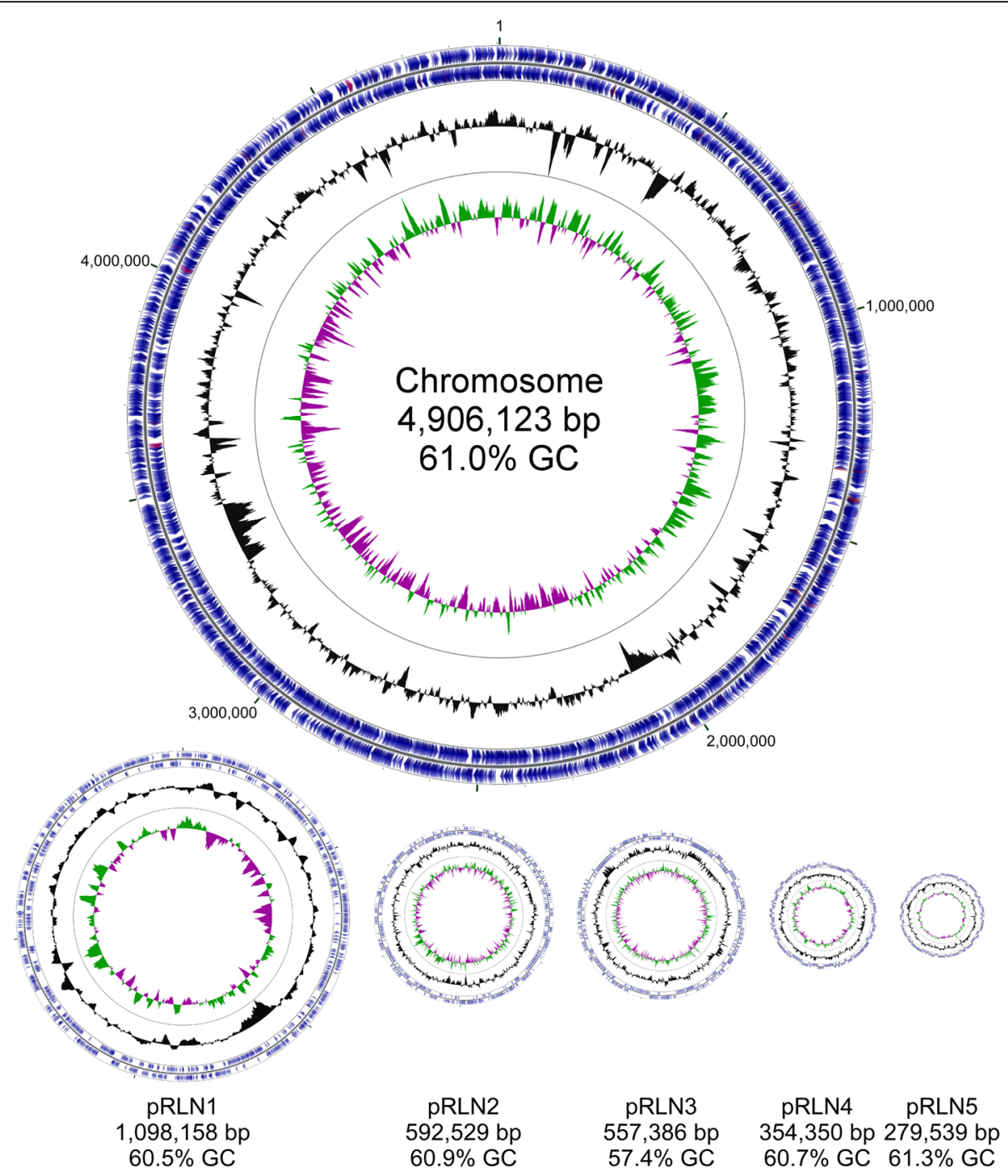

Fig. 3 The chromosome and five plasmids of R/ Norway. The plasmids are depicted to scale with the chromosome one-half of this scale. The outermost circles show protein encoding genes (blue) and rRNA and tRNA genes (red) in clockwise and counter-clockwise orientation. The inner circles indicate deviations in GC content (black) and GC skew (green/purple). Plasmid maps were generated using GCView [61]

lies in the number of not functionally classifiable genes (1760 vs. 1158 genes) (Table 4).

\section{Plasmid repertoire and genospecies classification}

The five plasmids contain one set of putative rep $A B C$ replication system genes each [26]. Comparative analysis of the Rep proteins from $R l$ Norway with those from $R l v$ 3841 revealed high identity between plasmids pRLN1 and pRL12, between pRLN2 and pRL11, and between pRLN5 and pRL10 (Fig. 4a). Gene content comparison and synteny analysis supported this result. Although large portions of pRLN4 and pRL9 are similar (Fig. 4b, and $c$ ), the RepABC proteins encoded in pRLN4 are more similar to their orthologs in pR132503.

Plasmid pRLN3 is slightly different than the other replicons of $R l$ Norway (Additional file 3: Figure S2A). It does not exhibit significant similarity to Rlv 3841 (Fig. $4 \mathrm{~b}$, and c), has a slightly lower GC content and a lower proportion of protein encoding sequences (Additional file 4: Table S2), and has a higher proportion of putative encoded proteins without known homologs (Additional file 3: Figure S2A). In addition, it is the only plasmid containing potentially active transposons ( 2 copies) and several incomplete and therefore most likely inactivated transposon copies. The pRLN3 RepABC proteins share high similarity to their orthologs in pRL1.

For genospecies classification, we compared the $R l$ Norway genome to representatives of the five proposed genospecies (gsA-gsE) [13]. Typically, genomes are regarded to belong to the same species if the ANI values are above $95 \%$. The two highest average nucleotide identity (ANI) scores ( $R l$ CC278f: 96.34\%; $R l$ SM51: 95.59\%) 
Table 3 Genome statistics for R/ Norway

\begin{tabular}{llc}
\hline Attribute & Value & \%of Total \\
\hline Genome size (bp) & $7,788,085$ & 100.00 \\
DNA coding (bp) & $6,859,686$ & 88.08 \\
DNA G + C (bp) & $4,659,466$ & 59.83 \\
DNA scaffolds & 6 & 100.00 \\
Total genes & 8079 & 100.00 \\
Protein coding genes & 7866 & 97.36 \\
RNA genes & 73 & 0.90 \\
Pseudo genes & 150 & 1.86 \\
Genes in internal clusters & Not determined & Not determined \\
Genes with function prediction & 6147 & 76.09 \\
Genes assigned to COGs & 6106 & 75.58 \\
Genes with Pfam domains & 6295 & 77.92 \\
Genes with signal peptides & 619 & 7.66 \\
Genes with transmembrane helices & 1656 & 20.50 \\
CRISPR repeats & 0 & 0.00 \\
\hline
\end{tabular}

were found with members of the genospecies gsD. All other comparisons resulted in ANI scores below 95\% (Table 5). The ANI score between $R l$ Norway and $R l v$ 3841 , which belongs to gsB, is only $93.26 \%$. Although genospecies gsA and $R l C C 278 \mathrm{f}$ in gsD are not yet well supported [13], the results indicate that $R l$ Norway belongs to genospecies gsD. This also fits well with $R l$ Norway having a plasmid subtype combination typical for gsD strains ([13]\& personal communication Peter Young).

\section{Central metabolism}

In terms of central metabolic genes $R l$ Norway resembles $R l v$ 3841. Both strains harbour genes encoding enzymes of the tricarboxylic acid (TCA) cycle required for aerobic respiration and energy production [27], of the pentose phosphate pathway required for the oxidation of glucose and the synthesis of nucleotides [28], and of the Entner-Doudoroff pathway for the catabolism of glucose to pyruvate [29]. Both strains lack a gene encoding the phosphofructokinase, an essential enzyme of the Embden-Meyerhof-Parnas glycolysis. These genetic similarities were reflected in a similar growth

Table 4 Number of genes associated with general COG functional categories

\begin{tabular}{|c|c|c|c|}
\hline Code & Value & \%age & Description \\
\hline J & 210 & 2.67 & Translation, ribosomal structure and biogenesis \\
\hline A & 0 & 0 & RNA processing and modification \\
\hline K & 686 & 8.72 & Transcription \\
\hline L & 219 & 2.78 & Replication, recombination and repair \\
\hline B & 2 & 0.03 & Chromatin structure and dynamics \\
\hline $\mathrm{D}$ & 40 & 0.51 & Cell cycle control, Cell division, chromosome partitioning \\
\hline V & 74 & 0.94 & Defense mechanisms \\
\hline $\mathrm{T}$ & 415 & 5.28 & Signal transduction mechanisms \\
\hline M & 334 & 4.25 & Cell wall/membrane biogenesis \\
\hline N & 92 & 1.17 & Cell motility \\
\hline$U$ & 106 & 1.35 & Intracellular trafficking and secretion \\
\hline $\mathrm{O}$ & 199 & 2.53 & Posttranslational modification, protein turnover, chaperones \\
\hline C & 342 & 4.35 & Energy production and conversion \\
\hline G & 709 & 9.01 & Carbohydrate transport and metabolism \\
\hline E & 831 & 10.56 & Amino acid transport and metabolism \\
\hline $\mathrm{F}$ & 117 & 1.49 & Nucleotide transport and metabolism \\
\hline $\mathrm{H}$ & 210 & 2.67 & Coenzyme transport and metabolism \\
\hline I & 270 & 3.43 & Lipid transport and metabolism \\
\hline P & 318 & 4.04 & Inorganic ion transport and metabolism \\
\hline Q & 206 & 2.62 & Secondary metabolites biosynthesis, transport and catabolism \\
\hline $\mathrm{R}$ & 905 & 11.51 & General function prediction only \\
\hline S & 630 & 8.01 & Function unknown \\
\hline- & 1760 & 22.37 & Not in COGs \\
\hline
\end{tabular}

The total is based on the total number of protein coding genes in the genome 

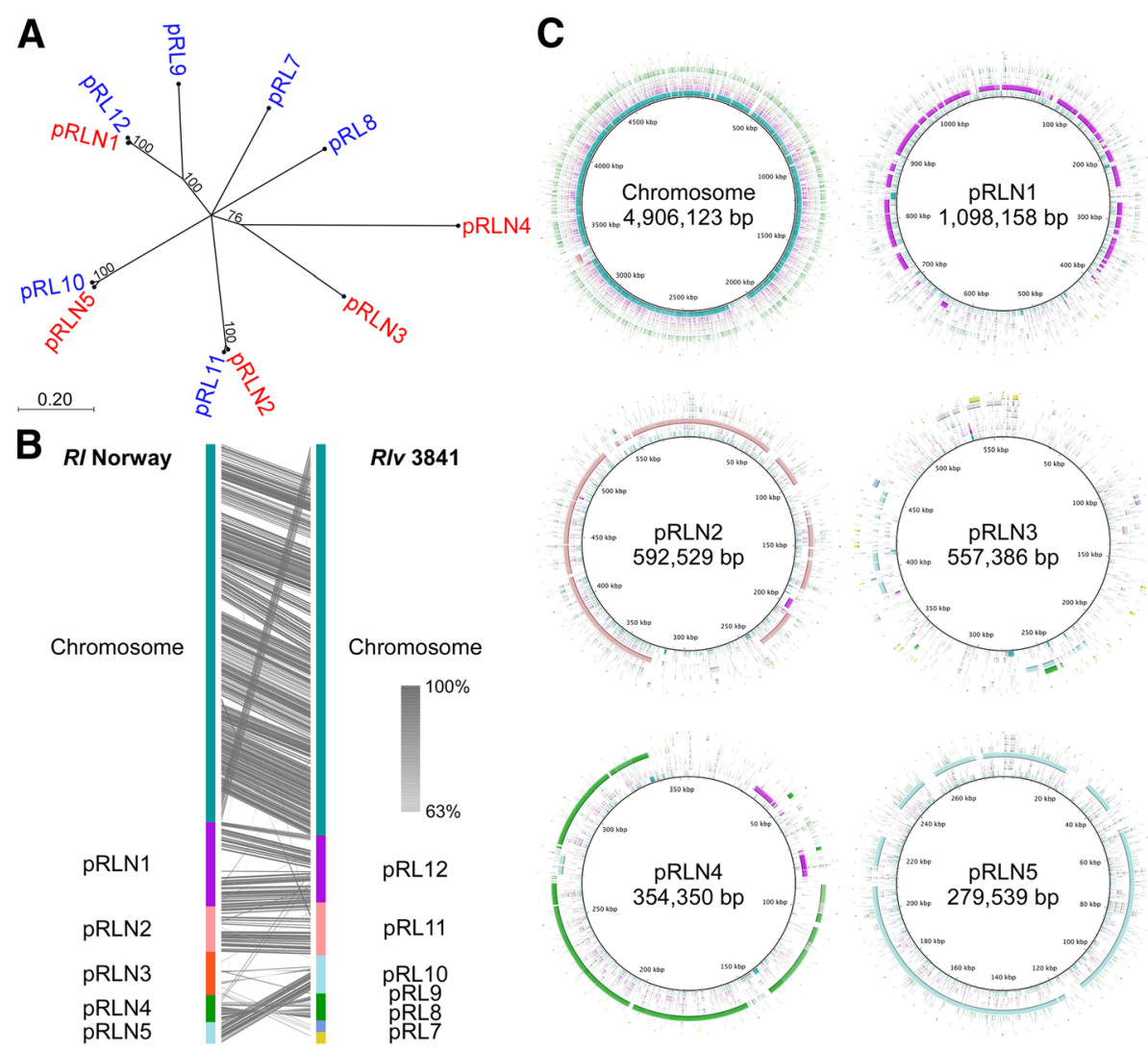

RIv 3841

Chromosome

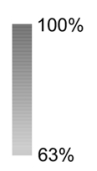

pRL12

pRL11

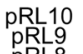

PRL9
pRL

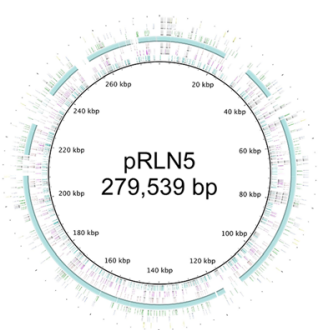

Fig. 4 Genome comparison between R/ Norway and R/v 3841. a Neighbor-joining tree of Rep proteins from both strains. Protein sequences for $\operatorname{Rep} A, \operatorname{Rep} B$, and $\operatorname{RepC}$ from the individual plasmids were aligned and the resulting alignments concatenated for analysis. RI Norway proteins are depicted in red, R/v3841 proteins in blue. Bootstrap values indicated on the nodes strongly support the relations between pRLN2 - pRL11, pRLN5 - pRL10, and pRLN1 - pRL12. Only bootstrap values $>70 \%$ are depicted. Branch lengths are given in terms of expected numbers of substitutions per nucleotide site. $\mathbf{b}$ For whole genome comparison the sequences of the chromosome and plasmids were concatenated for R/ Norway and R/V 3841 and compared with BlastN in Easyfig 2.2.2 [62]. Levels of sequence identity are indicated by different shades of grey. c Gene contents comparison between the two strains. Depicted are the $R /$ Norway replicons and their respective homologous regions from the R/V 3841 replicons. Plasmid maps were generated using BRIG [63]. Colors in the rings are the same as for the RIv 3841 replicons in (b)

pattern in different carbon sources using Biolog GN2 MicroPlates (Additional file 1: Figure S1) [13].

A noticeable difference in the Biolog assay was the assimilation of amino acids such as D- and L-alanine, L-serine and L-proline, and nucleosides. However, no major differences were observed in the genes mediating their metabolism. The only clear exceptions were that $R l$

Table 5 Genome comparison of R/ Norway with members of the five genospecies and the respective ANI scores

\begin{tabular}{lllll}
\hline & Norway vs & One-way ANI 1 & One-way ANI 2 & Two-way ANI \\
\hline (gsA) & WSM1325 & $93.45 \%$ & $93.52 \%$ & $93.70 \%$ \\
gsB & 3841 & $93.01 \%$ & $93.06 \%$ & $93.26 \%$ \\
gSC & TA1 & $93.75 \%$ & $93.80 \%$ & $93.94 \%$ \\
gsD & SM51 & $95.40 \%$ & $95.40 \%$ & $95.59 \%$ \\
(gsD) & CC278f & $96.11 \%$ & $96.19 \%$ & $96.34 \%$ \\
gsE & $128 C 53$ & $94.66 \%$ & $94.75 \%$ & $94.84 \%$ \\
\hline
\end{tabular}

Norway lacks a putative D-serine deaminase required for the conversion of D-serine to pyruvate, but contains two putative aspartate ammonia-lyases (CUJ84 pRLN3000095, CUJ84_pRLN3000303) and two putative asparagine synthetases (CUJ84_pRLN3000485, CUJ84_pRLN3000155). In terms of amino acid transport, two ABC-type broad specificity amino-acid transporters have been characterized in $R l v$ 3841, Aap (AapJQMP) and Bra (BraDEFGC) [30]. The bra (CUJ84_ Chr003782-3787) and aap (CUJ84_Chr001810-1813) clusters are highly conserved in $R l$ Norway. Another interesting difference concerned the metabolism of butanoate. In contrast to $R l v 3841, R l$ Norway did not grow on $\gamma$-hydroxybutyric acid (Additional file 1: Figure S1). This is supported by the lack of a gene cluster (pRL100133138 in $R l v$ 3841) associated to $\gamma$-hydroxybutyrate utilisation [13]. Furthermore, $R l$ Norway harbours an ortholog to the $p h b C 1$ gene (CUJ84_Chr001779), but lacks phbC2. 
These genes encode type I and type III poly- $\beta$-hydroxybutyrate (PHB) synthases, which are required for free-living and bacteroid PHB biosynthesis, respectively [31].

\section{Secretion systems}

Gram-negative bacteria secrete a suite of proteins via macromolecular complexes that have been classified as type 1-6 secretion systems in addition to the $s e c$ and tat transport systems [32]. A survey of the $R l$ Norway genome indicates that this strain contains a large repertoire of secretion systems that is distinct from the repertoire of $R l v 3841$ (Table 6). $R l$ Norway harbours five putative type 1 secretion systems (T1SS; Table 6). T1SSa, T1SSb and T1SSc are unique to $R l$ Norway. Interestingly, the genes encoding the T1SSa and T1SSc systems form operons with two large genes encoding putative repeats-in-

Table 6 Secretion system repertoire in R/ Norway

\begin{tabular}{|c|c|c|}
\hline Secretion system & Location & Mandatory genes (gene identifier) \\
\hline \multicolumn{3}{|c|}{ Type I secretion system (T1SS) } \\
\hline T1SSa & Chromosome & $\begin{array}{l}\text { hlyD (CUJ84_Chr000199), } \\
\text { hlyB (CUJ84_Chr000200) }\end{array}$ \\
\hline T1SSb & Chromosome & $\begin{array}{l}\text { hlyD (CUJ84_Chro00279), } \\
\text { hlyB (CUJ84_Chr000280) }\end{array}$ \\
\hline T1SSC & Chromosome & $\begin{array}{l}\text { hlyD (CUJ84_Chr002330), } \\
\text { hlyB (CUJ84_Chr002331) }\end{array}$ \\
\hline T1Ssd & Chromosome & $\begin{array}{l}\text { prsE (CUJ84_Chr003677), } \\
\text { prsD (CUJ84_Chr003678) }\end{array}$ \\
\hline T1Sse & Chromosome & $\begin{array}{l}\text { hlyD (CUJ84_Chr004833), } \\
\text { hlyB (CUJ84_Chr004834) }\end{array}$ \\
\hline T4SSa & pRLN1 & $\begin{array}{l}\text { virB1 (CUJ84_pRLN1000390), } \\
\text { virB2 (CUJ84_pRLN1000391), } \\
\text { virB3 (CUJ84_pRLN1000392), } \\
\text { virB4 (CUJ84_pRLN1000393), } \\
\text { virB5 (CUJ84_pRLN1000394), } \\
\text { virB6 (CUJ84_pRLN1000396), } \\
\text { virB8 (CUJ84_pRLN1000398), } \\
\text { virB9 (CUJ84_pRLN1000399), } \\
\text { virB10 (CUJ84_pRLN1000400) }\end{array}$ \\
\hline \multicolumn{3}{|c|}{ Type 5 secretion system (T5SS) } \\
\hline T5SSa & Chromosome & autB (CUJ84_Chr000739) \\
\hline T5SSb & Chromosome & Partial autB (CUJ84_Chr002323) \\
\hline T5SSC & pRLN2 & $\begin{array}{l}\text { tpsA (CUJ84_pRLN2000298), } \\
\text { tpsB (CUJ84_pRLN2000297) }\end{array}$ \\
\hline \multicolumn{3}{|c|}{ Type 6 secretion system (T6SS) } \\
\hline T6SS & pRLN1 & $\begin{array}{l}\text { tssB (CUJ84_pRLN1000762), } \\
\text { tssC (CUJ84_pRLN1000760, } \\
\text { CUJ84_pRLN1000761), } \\
\text { tssD (CUJ84_pRLN1000765), } \\
\text { tssE (CUJ84_pRLN1000758), } \\
\text { tssF (CUJ84_pRLN1000757), } \\
\text { tssG (CUJ84_pRLN1000756), } \\
\text { tssH (CUJ84_pRLN1000764), } \\
\text { tssl (CUJ84_pRLN1000767), } \\
\text { tssK (CUJ84_pRLN1000754), } \\
\text { tssL (CUJ84_pRLN1000753), } \\
\text { tssM (CUJ84_pRLN1000752) }\end{array}$ \\
\hline
\end{tabular}

toxin (RTX) toxins. The proteins forming the T1SSd and T1SSe have orthologs with more than $90 \%$ identity in $R l v$ 3841. For instance, the T1SSd proteins are orthologous to the PrsD and PrsE proteins of Rlv 3841 that are required for biofilm formation [33]. Like $R l v$ 3841, $R l$ Norway lacks T2SS and T3SS, but harbours T4SS and T6SS [34].

Bacteria utilize T3SS, T4SS and/or T6SS to inject effector proteins directly into eukaryotic host cells or into other bacteria [35-37]. In rhizobia, these effectors can mediate compatibility with the host [38]. $R l$ Norway harbours a putative T4SS that is distinct from the T4SS from $R l v$ 3841. The respective T4SS encoding virB operons are not syntenic and the encoding genes share on average less than $30 \%$ identity. The T4SS of $R l$ Norway is encoded in the pRLN1 plasmid and is predicted to translocate proteins and not DNA, as $R l$ Norway lacks a VirD2 relaxase [39]. In addition, it has the peculiarity that the virB11 gene is partially duplicated and two genes are located in-between the duplication.

$R l$ Norway and $R l v 3841$ harbour syntenic imp (tss) and $h c p$ clusters encoding type (i) T6SS. In both cases the imp cluster is lacking orthologs to the evpJ and tssJ genes. However, a comparison to Agrobacterium tumefaciens C58 revealed that these genes are also absent in the corresponding imp and $h c p$ operons (atu4330-atu4352). In addition, all essential genes for protein secretion are conserved [40].

T5SS are structures in which the cargo protein translocates itself across the plasma membrane. These are classified into auto-transporters (translocator and cargo encoded in the same gene) and two-partner systems (translocator and cargo are encoded by two separate genes) [41]. $R l$ Norway harbours two T5SS auto-transporters. However, T5SSb is split into two genes and it is probably not a bona fide T5SS. $R l$ Norway also has one two-partner system, in which the cargo protein is a putative filamentous hemagglutinin (Table 6). In contrast, Rlv 3841 contains three auto-transporters, but no two-partner system [34].

\section{Symbiotic gene repertoire}

Plasmid pRLN3 harbours all symbiotic genes in $R l$ Norway. The nod genes that are required for the synthesis and export of the nodulation factor, a key determinant in compatibility, are organised in one cluster (CUJ84. pRLN3000416-426) comprising the nodJICBADFELMN genes. They have the same organisation as the nod cluster in $R l v 3841$ [24], and the encoded proteins share at least 93.6\% identity with their Rlv 3841 orthologs. However, in contrast to $R l v$ 3841, $R l$ Norway lacks nodO and nodT orthologs in the proximity of the nod cluster. Interestingly, genes encoding putative transposases flank the $R l$ Norway nod cluster. The genes required for nitrogen fixation are 
located in proximity. The fixABCX (CU)84_pRLN3000397400) and the nifAB genes (CUJ84_pRLN3000401-402) are located almost directly downstream nodJ, whereas nifNEKDH (CUJ84_pRLN3000271-275), fixSIHG (CUJ84_ pRLN3000258-261) and fixPQON (CUJ84_pRLN3000263266) are located approximately $137.5 \mathrm{~kb}$ downstream of nodJ. The three subunits of the nitrogenase encoded by the nifHDK genes share 99.7, 93.5, and 96.3\% identity to their respective Rlv 3841 orthologs. A noteworthy difference between both strains is that $R l$ Norway harbours a single fixNOQP operon encoding the essential $\mathrm{cbb}_{3}$ terminal oxidase, whereas $R l v 3841$ contains two copies [24]. Furthermore, $R l$ Norway lacks genes encoding the FixK and FixL transcriptional regulators, which together with FnrN control the expression of the nitrogen fixation genes in other rhizobia strains [42]. Instead, $R l$ Norway harbours two putative furN genes (CUJ84_Chr002641, CUJ84_pRLN3000544) that are located in the chromosome and in the pRLN3 symbiotic plasmid. This is reminiscent of $R$. leguminosarum bv. viciae UPM791, in which FnrN is the global regulator of the fix genes. In this strain, FnrN is regulated by micro-aerobic conditions and binds a palindromic element called anaerobox $[43,44]$. Putative anaerobox sequences were found upstream of furN1 (CUJ84_Chr002641) and the fixNOQP and fixGHIS operons, which suggest that FnrN might regulate their expression in $R l$ Norway. However, no anaerobox was found upstream of furN2 (CUJ84_pRLN3000544). Interestingly, fnrN2 is approximately $16.5 \mathrm{~kb}$ upstream of a putative uptake hydrogenase cluster comprising 18 genes (CUJ84_ pRLN3000511-528). The cluster organisation resembles the hup and hyp genes from Rlv UPM791 [45]. Notably, $R l v 3841$ lacks such a hydrogenase cluster.

\section{Conclusions}

Although detrimental in agriculture, ineffective nitrogen-fixing symbiosis remains poorly investigated. In this regard, $R l$ Norway is an interesting strain as it exhibits a parasitic behaviour in a wide range of hosts. Comparative genomic analyses with other $R$. leguminosarum strains have the potential to reveal novel factors mediating symbiotic compatibility and efficiency.

\section{Additional files}

Additional file 1: Figure S1. $R /$ Norway substrate utilization pattern determined by Biolog. In blue and yellow are indicated substrates only utilized by $R /$ Norway and R/v 3841, respectively. Green indicates substrates used by both strains, whereas white depicts conditions in which both strains did not grow. R/v 3841 utilization pattern was extracted from [1]. (TIF $9702 \mathrm{~kb}$ )

Additional file 2: Table S1. Nodulation phenotypes of R/ Norway on selected hosts. (DOCX $68 \mathrm{~kb}$ )

Additional file 3: Figure S2. Distribution of functional classes of protein encoding genes within the RI Norway genome. (A) Functional class distribution across the six R/ Norway replicons. (B) Comparison of the relative occurrence of functionally classified protein encoding genes between the R/ Norway and R/V 3841 genomes. Functional annotation (COG) was performed on WebMGA server [1]. (TIF 10046 kb)

Additional file 4: Table S2. Genome statistics for R/ Norway. (DOCX $47 \mathrm{~kb}$ )

\section{Abbreviations}

COGs: Clusters of orthologous groups; CTAB: Cetyl trimethylammonium bromide; DAMPs: Damage associated molecular patterns; PHB: Poly- $\beta-$ hydroxybutyrate; Rl: Rhizobium leguminosarum; RTX: Repeats-in-toxin;

T1SS: Type 1 secretion system; TCA: Tricarboxylic acid

\section{Acknowledgements}

We thank Martin Parniske for providing the R/ Norway strain and critical reading of the manuscript, Moritz Thomas for expert technical assistance in MinION library preparation and sequencing, and Marion Cerri for insightful discussions and reading the manuscript. We specially thank Peter Young for sharing unpublished sequences and fruitful discussions.

\section{Funding}

This work was funded by the German research foundation (DFG-grant: MA7269-1).

\section{Authors' contributions}

$J \mathrm{l}$ performed the imaging, the chemotaxonomic analyses, and extracted the genomic DNA. AB conducted the genome sequencing and annotation. $\mathrm{AH}$ conducted the genome assembly and comparisons. MM conducted the phylogenetic analysis and the manual inspection of the annotation. MM and $A B$ conceived the experiments and wrote the manuscript. All authors read and approved the final manuscript.

\section{Competing interests}

The authors declare that they have no competing interests.

\section{Publisher's Note}

Springer Nature remains neutral with regard to jurisdictional claims in published maps and institutional affiliations.

Received: 9 February 2018 Accepted: 10 November 2018

Published online: 05 December 2018

References

1. Stagnari F, Maggio A, Galieni A, Pisante M. Multiple benefits of legumes for agriculture sustainability: an overview. Chem Biol Technol Agric. 2017;4(1):2.

2. Rubiales $D$, Mikic A. Introduction: legumes in sustainable agriculture. Critl Rev Plant Sci. 2015:34(1-3):2-3.

3. Oldroyd GE, Murray JD, Poole PS, Downie JA. The rules of engagement in the legume-rhizobial symbiosis. Annu Rev Genet. 2011:45:119-44.

4. Westhoek A, Field E, Rehling F, Mulley G, Webb I, Poole PS, Turnbull LA. Policing the legume-Rhizobium symbiosis: a critical test of partner choice. Sci Rep. 2017;7(1):1419.

5. Streeter JG. Failure of inoculant rhizobia to overcome the dominance of indigenous strains for nodule formation. Can J Microbiol. 1994;40(7):513-22.

6. Parker DT, Allen ON. The nodulation status of Trifolium ambiguum. Soil Sci Soc Am Pro. 1952;16(4):350-3.

7. Howieson JG, Yates RJ, O'Hara GW, Ryder M, Real D. The interactions of Rhizobium leguminosarum biovar trifolii in nodulation of annual and perennial Trifolium spp. from diverse centres of origin. Aust J Exp Agric. 2005:45(3):199-207.

8. Amesgottfred NP, Christie BR. Competition among strains of Rhizobium leguminosarum biovar trifolii and use of a diallel analysis in assessing competition. Appl Environ Microb. 1989;55(6):1599-604.

9. Winarno R, Lie TA. Competition between Rhizobium strains in nodule formation - interaction between nodulating and non-nodulating strains. Plant Soil. 1979;51(1):135-42.

10. Bohlool BB, Ladha JK, Garrity DP, George T. Biological nitrogen fixation for sustainable agriculture - a perspective. Plant Soil. 1992;141(1-2):1-11.

11. Fred EB, Baldwin IL, McCoy E. Root nodule bacteria and leguminous plants; 1932. 
12. Gossmann JA, Markmann K, Brachmann A, Rose LE, Parniske M. Polymorphic infection and organogenesis patterns induced by a Rhizobium leguminosarum isolate from Lotus root nodules are determined by the host genotype. New Phytol. 2012;196(2):561-73.

13. Kumar N, Lad G, Giuntini E, Kaye ME, Udomwong P, Shamsani NJ, Young JPW, Bailly X. Bacterial genospecies that are not ecologically coherent: population genomics of Rhizobium leguminosarum. Open Biol. 2015;5(1):140133.

14. Murray MG, Thompson WF. Rapid isolation of high molecular-weight plant DNA. Nucleic Acids Res. 1980;8(19):4321-5.

15. Wick RR, Judd LM, Gorrie CL, Holt KE. Unicycler: Resolving bacterial genome assemblies from short and long sequencing reads. PLOS Comput Biol. 2017; 13(6):e1005595.

16. Overbeek R, Olson R, Pusch GD, Olsen GJ, Davis JJ, Disz T, Edwards RA, Gerdes S, Parrello B, Shukla M, et al. The SEED and the rapid annotation of microbial genomes using subsystems technology (RAST). Nucleic Acids Res. 2014;42(D1):D206-14.

17. Aziz RK, Bartels D, Best AA, DeJongh M, Disz T, Edwards RA, Formsma K, Gerdes S, Glass EM, Kubal M, et al. The RAST server: rapid annotations using subsystems technology. BMC Genomics. 2008;9(1):75.

18. Vallenet D, Calteau A, Cruveiller S, Gachet M, Lajus A, Josso A, Mercier J, Renaux A, Rollin J, Rouy Z, et al. MicroScope in 2017: an expanding and evolving integrated resource for community expertise of microbial genomes. Nucleic Acids Res. 2017;45(D1):D517-28.

19. Tatusov RL, Koonin EV, Lipman DJ. A genomic perspective on protein families. Science. 1997;278:631-7.

20. Petersen TN, Brunak S, von Heijne G, Nielsen H. SignalP 4.0: discriminating signal peptides from transmembrane regions. Nat Methods. 2011;8:785.

21. Finn RD, Coggill P, Eberhardt RY, Eddy SR, Mistry J, Mitchell AL, Potter SC, Punta M, Qureshi M, Sangrador-Vegas A, et al. The Pfam protein families database: towards a more sustainable future. Nucleic Acids Res. 2016;44(D1): D279-85.

22. Krogh A, Larsson B, von Heijne G, Sonnhammer EL. Predicting transmembrane protein topology with a hidden Markov model: application to complete genomes. J Mol Biol. 2001;305:567-80.

23. Grissa I, Vergnaud G, Pourcel C. CRISPRFinder: a web tool to identify clustered regularly interspaced short palindromic repeats. Nuclein Acids Res. 2007;35:W52-7.

24. Young JP, Crossman LC, Johnston AW, Thomson NR, Ghazoui ZF, Hull KH, Wexler M, Curson AR, Todd JD, Poole PS, et al. The genome of Rhizobium leguminosarum has recognizable core and accessory components. Genome Biol. 2006;7(4):R34

25. Simão FA, Waterhouse RM, loannidis $P$, Kriventseva EV, Zdobnov EM. BUSCO: assessing genome assembly and annotation completeness with single-copy orthologs. Bioinformatics. 2015;31(19):3210-2.

26. Ramírez-Romero MA, Soberón N, Pérez-Oseguera A, Téllez-Sosa J, Cevallos MA. Structural elements required for replication and incompatibility of the Rhizobium etli symbiotic plasmid. J Bacteriol. 2000;182(11):3117-24.

27. Dunn MF. Tricarboxylic acid cycle and anaplerotic enzymes in rhizobia. FEMS Microbiol Rev. 1998;22(2):105-23.

28. Stincone A, Prigione A, Cramer T, Wamelink MMC, Campbell K, Cheung E, Olin-Sandoval V, Gruning NM, Kruger A, Alam MT, et al. The return of metabolism: biochemistry and physiology of the pentose phosphate pathway. Biol Rev. 2015;90(3):927-63.

29. Conway T. The Entner-Doudoroff pathway - history, physiology and molecular biology. FEMS Microbiol Lett. 1992;103(1):1-28.

30. Hosie AHF, Allaway D, Galloway CS, Dunsby HA, Poole PS. Rhizobium leguminosarum has a second general amino acid permease with unusually broad substrate specificity and high similarity to branched-chain amino acid transporters (bra/LIV) of the ABC family. J Bacteriol. 2002;184(15):4071-80.

31. Terpolilli JJ, Masakapalli SK, Karunakaran R, Webb IUC, Green R, Watmough NJ, Kruger NJ, Ratcliffe RG, Poole PS. Lipogenesis and redox balance in nitrogen-fixing pea bacteroids. J Bacteriol. 2016;198(20):2864-75.

32. Green E, Mecsas J. Bacterial secretion systems: an overview. In: Kudva I, Cornick N, Plummer P, Zhang Q, Nicholson T, Bannantine J, Bellaire B, editors. Virulence mechanisms of bacterial pathogens, Fifth Edition. Washington, DC: ASM Press; 2016. p. 215-39. https://doi.org/10.1128/ microbiolspec.VMBF-0012-2015.

33. Russo DM, Williams A, Edwards A, Posadas DM, Finnie C, Dankert M, Downie JA, Zorreguieta A. Proteins exported via the PrsD-PrsE type I secretion system and the acidic exopolysaccharide are involved in biofilm formation by Rhizobium leguminosarum. J Bacteriol. 2006;188(12):4474-86.

34. Krehenbrink M, Downie JA. Identification of protein secretion systems and novel secreted proteins in Rhizobium leguminosarum bv. viciae. BMC Genomics. 2008;9(1):55.

35. Cianfanelli FR, Monlezun L, Coulthurst SJ. Aim, load, fire: the type VI secretion system, a bacterial nanoweapon. Trends Microbiol. 2016;24(1): 51-62.

36. Ding ZY, Atmakuri K, Christie PJ. The outs and ins of bacterial type IV secretion substrates. Trends Microbiol. 2003;11(11):527-35.

37. Deng WY, Marshall NC, Rowland JL, McCoy JM, Worrall LJ, Santos AS, Strynadka NCJ, Finlay BB. Assembly, structure, function and regulation of type III secretion systems. Nat Rev Microbiol. 2017;15(6):323-37.

38. Nelson MS, Sadowsky MJ. Secretion systems and signal exchange between nitrogen-fixing rhizobia and legumes. Front Plant Sci. 2015;6:491.

39. Alvarez-Martinez CE, Christie PJ. Biological diversity of prokaryotic type IV secretion systems. Microbiol Mol Biol Rev. 2009;73(4):775-808.

40. Ma LS, Hachani A, Lin JS, Filloux A, Lai EM. Agrobacterium tumefaciens deploys a superfamily of type $\mathrm{VI}$ secretion DNase effectors as weapons for interbacterial competition in planta. Cell Host Microbe. 2014;16(1): 94-104.

41. Abby SS, Cury J, Guglielmini J, Neron B, Touchon M, Rocha EP. Identification of protein secretion systems in bacterial genomes. Sci Rep. 2016;6:23080.

42. Fischer HM. Genetic regulation of nitrogen-fixation in rhizobia. Microbiol Rev. 1994;58(3):352-86.

43. Gutiérrez D, Hernando Y, Palacios JM, Imperial J, Ruiz-Argüeso T. FnrN controls symbiotic nitrogen fixation and hydrogenase activities in Rhizobium leguminosarum bv. viciae UPM791. J Bacteriol. 1997;179(17):5264-70.

44. Colombo MV, Gutiérrez D, Palacios JM, Imperial J, Ruiz-Argüeso T. A novel autoregulation mechanism of fnrN expression in Rhizobium leguminosarum bv. viciae. Mol Microbiol. 2000;36(2):477-86.

45. Baginsky C, Brito B, Imperial J, Palacios JM, Ruiz-Argüeso T. Diversity and evolution of hydrogenase systems in rhizobia. Appl Environ Microbiol. 2002; 68(10):4915-24.

46. Field D, Garrity G, Gray T, Morrison N, Selengut J, Sterk P, Tatusova T, Thomson N, Allen MJ, Angiuoli SV, et al. The minimum information about a genome sequence (MIGS) specification. Nat Biotechnol. 2008;26(5):541-7.

47. Field D, Amaral-Zettler L, Cochrane G, Cole JR, Dawyndt P, Garrity GM Gilbert J, Glockner FO, Hirschman L, Karsch-Mizrachi I, et al. The genomic standards consortium. PLoS Biol. 2011:9(6):e1001088.

48. Woese CR, Kandler O, Wheelis ML. Towards a natural system of organisms: proposal for the domains archaea, Bacteria, and Eucarya. PNAS. 1990;87(12): 4576-9.

49. Garrity GM, Bell JA, Lilburn T, Phylum XIV. Proteobacteria phyl. nov., vol. 2 Part B, 2 edn. New York: Springer; 2005.

50. Euzéby J. Validation list no. 107. List of new names and new combinations previously effectively, but not validly, published. Int I Syst Evol Microbiol. 2006;56:1-6.

51. Garrity GM, Bell JA, Lilburn T, Class I. Alphaproteobacteria class. nov., vol. 2 Part C. 2nd ed. New York: Springer; 2005.

52. Kuykendall LD. Order VI. Rhizobiales ord. nov. In: Bergey's Manual of Systematic Bacteriology. Edited by Garrity GM, Brenner DJ, Kreig NR, Staley IT, vol. 2, Part C, 2 edn. New York: Springer; 2005: 324

53. Kuykendall LD. Rhizobiaceae. In: Garrity GM, Brenner DJ, Kreig NR, Staley JT, editors. Bergey's Manual of Systematic Bacteriology. New York: Springer; 2005

54. Conn HJ. Taxonomic relationships of certain non-sporeforming rods in soil. J Bacteriol. 1938;36:320-1.

55. Skerman VBD, Mcgowan V, Sneath PHA. Approved lists of bacterial names. Int J Syst Bacteriol. 1980;30(1):225-420.

56. Kuykendall LD, J.M. Y, Martínez-Romero E, Kerr A, Sawada H. Rhizobium. In: Bergey's manual of systematic bacteriology. Edited by Garrity GM, Brenner DJ, Kreig NR, Staley JT. New York: Springer; 2005

57. Frank B. Über die Pilzsymbiose der Leguminosen. Ber Dtsch Bot Ges. 1889;7: 332-46.

58. Young JM, Kuykendall LD, Martinez-Romero E, Kerr A, Sawada H. A revision of Rhizobium frank 1889, with an emended description of the genus, and the inclusion of all species of Agrobacterium conn 1942 and Allorhizobium undicola de Lajudie et al. 1998 as new combinations: Rhizobium radiobacter, R. rhizogenes, R. rubi, R. undicola and R. vitis. Int I Syst Evol Microbiol 2001, 51(Pt 1):89-103. 
59. Ramírez-Bahena MH, Garcia-Fraile P, Peix A, Valverde A, Rivas R, Igual JM, Mateos PF, Martinez-Molina E, Velazquez E. Revision of the taxonomic status of the species Rhizobium leguminosarum (frank 1879) frank 1889AL, Rhizobium phaseoli Dangeard 1926AL and Rhizobium trifolii Dangeard 1926AL. R. trifolii is a later synonym of $R$. leguminosarum. Reclassification of the strain R. leguminosarum DSM 30132 (=NCIMB 11478) as Rhizobium pisi sp. nov. Int J Syst Evol Microbiol. 2008;58(Pt 11):2484-90.

60. Ashburner M, Ball CA, Blake JA, Botstein D, Butler H, Cherry JM, Davis AP, Dolinski K, Dwight SS, Eppig JT, et al. Gene ontology: tool for the unification of biology. the Gene Ontology Consortium. Nat Genet. 2000;25(1):25-9.

61. Stothard P, Wishart DS. Circular genome visualization and exploration using CGView. Bioinformatics. 2005;21(4):537-9.

62. Sullivan MJ, Petty NK, Beatson SA. Easyfig: a genome comparison visualizer. Bioinformatics. 2011;27(7):1009-10.

63. Alikhan NF, Petty NK, Ben Zakour NL, Beatson SA. BLAST ring image generator (BRIG): simple prokaryote genome comparisons. BMC Genomics. 2011;12(1):1.

Ready to submit your research? Choose BMC and benefit from:

- fast, convenient online submission

- thorough peer review by experienced researchers in your field

- rapid publication on acceptance

- support for research data, including large and complex data types

- gold Open Access which fosters wider collaboration and increased citations

- maximum visibility for your research: over $100 \mathrm{M}$ website views per year

At $\mathrm{BMC}$, research is always in progress.

Learn more biomedcentral.com/submissions 